\title{
El cambio de la Look East Policy \\ a la Act East Policy en India ${ }^{1}$ \\ From the Look East Policy \\ to the Act East Policy in India
}

Paola Andrea Baroni ${ }^{2}$ y María Noel Dussort ${ }^{3}$

Resumen: La Look East Policy marcó la forma en que India se ha vinculado con el Sudeste Asiático desde 1992. Inicialmente conducida por intereses comerciales, su objeto se ha ampliado hacia la construcción de vínculos institucionales, económicos y de seguridad y defensa. La administración de Narendra Modi inaugurada en 2014 rebautizó esta orientación de política exterior como Act East Policy, propiciando una acción mayor.

Aplicando una metodología cualitativa, el objetivo de este trabajo es describir la forma en que la Look East Policy ha evolucionado hacia la Act East Policy y analizar las acciones llevadas a cabo por el nuevo gobierno indio para dilucidar si se evidencia una profundización en la relaciones con los países del Este de Asia.

Abstract: Look East Policy delimited the way India has established its relations with South East Asia since 1992. Initially, it was led by commercial interests but its purpose was extended towards institutional, economic and security and defense ties. Narendra Modi's administration renamed this approach as Act East Policy, backing a bigger action.

Through a qualitative methodology, the aim of this article is to describe how the Look East Policy has evolved to the Act East Policy and analyze the actions being accomplished by the new Indian government to elucidate if there is a deep change within the East Asia's countries relationship.

Palabras claves: India, Look East Policy, Act East Policy, Sudeste Asiático. Key words: India, Look East Policy, Act East Policy, South East Asia.

${ }^{1}$ Recibido: 24/10/2016. Aprobado: 28/04/2017

${ }^{2}$ Licenciada en Relaciones Internacionales de la Universidad Católica de Córdoba. Actualmente está cursando el Doctorado en Relaciones Internacionales de la Universidad Nacional de Rosario. Se especializa en Historia de las Relaciones Internacionales, Política Internacional, India, China y el Sudeste Asiático.

${ }^{3}$ Licenciada en Relaciones Internacionales y estudiante del Doctorado en Relaciones Internacionales de la Facultad de Ciencia Política y Relaciones Internacionales de la Universidad Nacional de Rosario (UNR). Becaria doctoral del Consejo Nacional de Investigaciones Científicas y Técnicas (CONICET). 


\section{Introducción}

El fin de la Guerra Fría marcó el comienzo de un proceso de descentralización global, caracterizado por el declive de Estados Unidos como superpotencia, la multipolaridad en diversas dimensiones del sistema internacional y la constitución de roles preponderantes de las llamadas potencias medias emergentes, entre ellas India. Estos cambios generaron una modificación en la distribución internacional del poder comenzando a darse un desplazamiento del mismo, de Occidente a Oriente.

Desde inicios de los años noventa, India ha desarrollado un estatus de potencia media que se observa en el incremento de sus capacidades materiales y de su influencia en el ámbito internacional. El primer punto se ejemplifica en su importante crecimiento económico, su capacidad militar, el desarrollo de poder nuclear, los progresos realizados en alta tecnología, la amplia fuerza laboral con la que cuenta y el impulso dado al sector servicios (Efstathopoulos, 2011).

Respecto a sus capacidades inmateriales, éstas se manifiestan a través del uso del llamado soft power o poder blando ${ }^{4}$, el cual se constituye en una herramienta importante ya que le permite ocupar posiciones de mayor relieve en términos de poder político, económico y social (Lara, 2012). Desde su independencia, los principios de la noalineación y la no interferencia han sido para India parte de sus herramientas discursivas de soft power buscando presentar una tercera vía entre Oriente y Occidente. Esta estrategia le ha permitido ganar cierta influencia a nivel global, aunque en la práctica estuvo constreñida por la rivalidad entre las superpotencias (Efstathopoulos, 2011).

Desde 1947 hasta la década del noventa la política exterior de India estuvo guiada por la necesidad de crear una economía autosuficiente y reducir los altos niveles de pobreza dentro de un marco democrático. Se diseñó, entonces, una estrategia socialista con fuerte intervención estatal, basada en el desarrollo de la industria pesada, una política de sustitución de importaciones y control financiero. El resultado de este modelo fue un excesivo control sobre la producción, la inversión y el comercio y políticas de estímulo al mercado interno, que sumieron al país en el aislamiento económico internacional (Rajan y Sen, 2000). Es por ello que a nivel económico-comercial India se mantuvo relativamente atrasada ya que contaba con una economía prácticamente cerrada al comercio exterior y a la inversión extranjera (BID, 2010).

Por otro lado, en los primeros años de vida independiente, hubo una explosión de actividades en búsqueda de revivir los antiguos lazos regionales resquebrajados por años de colonialismo británico. Tal es así que la llegada de Nehru al gobierno reactivó la política exterior hacia el Este. Así India apoyó los movimientos independentistas y los procesos de descolonización en el Sudeste Asiático (en adelante, SEA), actuando de acuerdo a su posición de no-alineamiento y a la crítica acérrima hacia al colonialismo (Gupta, 2007).

No obstante, el discurso sobre la construcción de una solidaridad asiática entre los países de la región -a finales de 1940 y principios de 1950- no se pudo llevar a la práctica debido al impacto de la Guerra Fría. A tal contexto se sumaban las tensas relaciones entre India y China (Mohapatra, 2014), y al hecho de que la región estaba menos des-

${ }^{4}$ El término poder blando fue acuñado por Joshep Nye (2004), quién lo define como la habilidad de obtener lo que se quiere a través de la atracción antes que por medios coercitivos o vía recompensas. Surge del atractivo de la cultura de un país, de sus ideales políticos y de sus políticas. 
arrollada que India hasta la década de 1980, por lo que el Sudeste Asiático no era un socio atractivo (Sikri, 2009). Es así como la dinámica propia del sistema internacional y las políticas económicas aislacionistas de Nueva Delhi reemplazaron aquel comienzo por una relación cada vez más distante con la región.

No obstante, entre 1970 y 1980 el Sudeste Asiático sufrió una gran transformación económica y comercial, a pesar de los conflictos y disputas regionales y las diferentes orientaciones políticas de los países que constituían la región. La preeminencia del factor económico por sobre las mencionadas diferencias llevó a un crecimiento económico acelerado que atrajo la atención de los principales Estados y regiones del mundo (Gupta, 2007), pasando a ser denominados nuevos países industrializados (NIC's por sus siglas en inglés).

En el caso de India, las condiciones internas de bajo crecimiento y baja productividad se conjugaron con las repercusiones que tuvo en la economía india la Guerra del Golfo de 1991, como fue el aumento del precio del petróleo. Esta situación llevó a la entrada en default de la economía india que condujo al país a aceptar un programa de ajuste estructural del Fondo Monetario Internacional (Rajan y Sen, 2000). La presión del momento se convirtió en el puntapié inicial para la liberalización y apertura económica y la consecuente inserción comercial internacional del país.

Esta situación condujo a India a reformular la política exterior y comercial, las cuales en adelante estarían guiadas por el interés nacional de crecimiento económico y desarrollo del país. Para llevar a cabo esta tarea, el gobierno indio impulsó el acercamiento hacia estados que puedan contribuir con este objetivo (Tharoor, 2012), y allí es donde los países del Sudeste Asiático ocuparon un rol importante. En este orden, India se acercó a la región con una postura más pragmática, utilizando el enfoque económico-comercial como principal conductor de su política exterior, con el fin de abrir mercados a sus productos y atraer inversiones (Gupta, 2007). Dentro de este contexto surgió en 1992 la Look East Policy (LEP), la cual fue expuesta por primera vez en 1994 y estuvo principalmente orientada a la Asociación de Naciones del Sudeste Asiático ${ }^{5}$.

\footnotetext{
${ }^{5}$ Para este trabajo se utilizará la sigla en inglés, ASEAN, para referirse a esta organización ya que es la más conocida y utilizada en el ámbito de las Relaciones Internacionales. La ASEAN fue fundada en 1967 a través de la firma de la Declaración de Bangkok, por los cinco miembros originales: Indonesia, Malasia, Filipinas, Singapur y Tailandia. Surgió luego de diversos intentos de organizaciones similares, como ASA (Asociación del Sureste Asiático) o ASPAC (Asia Pacific Council), recogiendo la reiterada voluntad de integración. En el marco de la Guerra Fría su origen respondió a la voluntad de evitar la expansión del comunismo desde Vietnam hacia los países vecinos y la existencia de múltiples conflictos territoriales que requerían de cierta institucionalidad para asegurar el uso de métodos pacíficos para su resolución. La ASEAN nació claramente como una iniciativa de cooperación regional pero a partir de los años setenta se dio un salto cualitativo hacia un verdadero proyecto de integración económica al que se incorporaron nuevos miembros: el Sultanado de Brunei (1984), Vietnam (1995), Laos y Myanmar (1997) y Camboya (1999).

En 1992, los temas de seguridad y de cooperación regional adquirieron una visión más amplia que condujo a la creación en 1994 del Foro Regional ASEAN (ARF, por sus siglas en inglés). El ARF se presentó como una nueva organización que agrupaba a 23 países en distintos estatus, mientras que en la actualidad se ha extendido a 27 países tanto regionales como extraregionales. Iniciado el siglo XXI, en la Declaración ASEAN Concord II de 2003, se produjo la definición de las áreas de interés de la organización, llamadas los "3 pilares" o las tres Comunidades: seguridad (ASEAN Security Community - ASC), economía (ASEAN Economic Community - AEC) y socio-cultural (ASEAN Socio Cultural Community - ASC), las cuales permitieron la incorporación de socios sectoriales no miembros de ASEAN. También se ha pro-
} 
Desde entonces, las relaciones con dicha Asociación han sido la puerta de entrada al Sudeste Asiático. El Primer Ministro Narasimha Rao fue quien lanzó el proyecto y puede afirmarse que la LEP es un claro ejemplo del realismo diplomático del Primer Ministro al proponer esta iniciativa en un momento de crisis nacional sobre el rumbo a seguir (Mohapatra, 2014). En ese sentido, el gobierno de Rao contaba con una fuerte determinación para jugar un rol central, adecuando su imagen al escenario internacional (Mohapatra, 2014) así como la intención de acercarse a los vecinos asiáticos (Gupta, 2007).

Danielle Rajendram (2014) ha identificado tres factores que llevaron al diseño de la Look East Policy. En primer lugar, el colapso de la Unión Soviética que creó un vacío económico y de poder para India ya que era su principal socio comercial y estratégico. En segundo lugar, la apertura de China durante la década de 1980 provocó en India la necesidad de acercarse al SEA para evitar caer en un rol subordinado a nivel político y económico en la región. En tercer lugar, el deseo de India de desarrollar y estabilizar sus estados del Noreste ${ }^{6}$, especialmente los que limitan con Myanmar, los cuales estaban en medio de un período de insurgencia. Por otra parte, la guerra del Golfo de 1991 marcó la necesidad de diversificar las fuentes de energía y los socios económico-comerciales (Gupta, 2007). Así, el objetivo de lograr un crecimiento económico sostenido y de abastecerlo con los recursos necesarios hizo del SEA una prioridad (Rajendram, 2014).

La ASEAN respondió favorablemente a la nueva política de India. Una vez que Nueva Delhi tomó la iniciativa de acercarse a la región, el crédito por profundizar las relaciones hay que dárselo a la propia Asociación, que fue el motor del desarrollo de las vinculaciones (Gupta, 2007). Más aún, antes de pronunciada la LEP, la ASEAN le otorgó a India el status de Socio de Diálogo Sectorial ${ }^{7}$ en 1992, y a partir de allí, el acercamiento se enfocó en cuatro áreas con gran potencial de cooperación, como ser comercio, inversiones, turismo y recursos para el desarrollo humano, incluyendo la cooperación en ciencia y tecnología (Mohapatra, 2014).

Al principio, tanto la LEP lanzada por India como el estatus de Socio de Diálogo Sectorial otorgado por la ASEAN a Nueva Delhi, no cambiaron de manera radical la actitud casi pasiva de India y por eso la ASEAN volvió a promover su status esperando que fuera más proactiva en el futuro. Es así que en 1995 se la ascendió a la categoría de Socio Pleno de Diálogo en la Cumbre de Tailandia. Gupta (2007) plantea que la decisión se comunicó a India en la reunión con la ASEAN -sin anticipación-, ya que fue

movido la vinculación con la República de Corea, Japón y la República Popular China, desarrollando un nuevo formato denominado ASEAN + 3.

${ }^{6}$ La región del Nordeste está compuesta por siete Estados: Mizoram, Meghalaya, Arunachal Pradesh, Nagalandia Assam, Manipur y Tripura. Los últimos cuatro estados son los principalmente afectados por la insurgencia, aunque toda la región en su conjunto ha sufrido desde la independencia episodios de violencia armada.

${ }^{7}$ En sus relaciones exteriores, la ASEAN busca desarrollar lazos de amistad y diálogo mutuamente beneficiosos, a través de la cooperación y asociación con países, organizaciones e instituciones sub-regionales, regionales e internacionales. Para llevar a cabo este objetivo, la ASEAN ha desarrollado ciertas categorías a otorgar a un actor no miembro de la organización, como ser la de Socio de Diálogo, Socio de Diálogo Sectorial, Socio de Desarrollo, Observador Especial, Invitado, u otros. Cada una de estas categorías hace referencia a un nivel diferente de vinculación con la organización. Información extraída de http://asean.org/asean/external-relations/ [Consultado el 11-05-2017]. 
tomada como consecuencia de la retórica y las acciones agresivas de China entre 199495 hacia la región ${ }^{8}$. En una próxima etapa, India fue aceptada como Socio a Nivel de Cumbre de la ASEAN en el año 2000 (Gupta, 2007). La importancia de los acuerdos negociados y las constantes interacciones generadas durante los últimos años entre la ASEAN e India han llevado a que en 2012 esta asociación haya alcanzado la cualidad de 'estratégica' (Rajendram, 2014).

Para los países de la ASEAN, India se presenta como una opción para balancear la gran dependencia económica de China y también contribuye a proveer y mantener el equilibrio y la estabilidad en la región (Rajendram, 2014). Debido a ello, la LEP se ha ido moviendo de los temas económicos-comerciales hacia una agenda más amplia, que incluye cooperación en materia de seguridad (Gupta, 2007).

Con la integración de India al Asia-Pacífico a través de la ASEAN, surgió la necesidad de expandir la LEP hasta incluir todo este ámbito geográfico. La primera fase (1994-2003) y la segunda fase (2004-2013) de la LEP estuvieron centradas en la ASEAN y enmarcadas en las vinculaciones comerciales y de inversiones. La llegada al gobierno de Narendra Modi en 2014 generó un nuevo impulso a la misma, denominándose ahora Act East Policy (AEP) -política de acción hacia el Este-, queriendo acentuar una política exterior y comercial más proactiva, en vez de una marcada por el idealismo, la retórica y la reacción. En otras palabras, con el cambio de LEP en AEP Modi pretendió dar un salto cualitativo en sus vínculos con los países del Este, para diferenciarse del gobierno anterior ${ }^{9}$.

Por lo tanto, esta tercera fase (2014-...), presenta una definición más amplia del 'Este', extendiéndose desde Australia y el Pacífico-Sur hasta el Este de Asia, con la ASEAN como centro. Esta etapa también se ha caracterizado por una apertura hacia otros temas, incluyendo esfuerzos conjuntos para lograr conectividad física con el SEA ${ }^{10}$, proteger las líneas marítimas y coordinar actividades contra el terrorismo.

Así, el nuevo gobierno del Bharatija Janata Party (BJP) ha puesto el ímpetu en fortalecer especialmente las relaciones con Japón, Vietnam, Australia y la ASEAN (Rajendram 2014). Para lograrlo, en sus dos primeros años de gobierno, el Primer Ministro Modi realizó visitas oficiales a Myanmar, Malasia y Singapur. El Presidente indio, Pranab Mukherjee, hizo lo propio en Vietnam y el Vicepresidente Hamid Ansari visitó Camboya, Laos e Indonesia. Por su parte, la Ministra de Relaciones Exteriores, Sushma Swaraj, realizó una gira por Myanmar, Vietnam, Tailandia, Singapur e Indonesia y recibió la visita de su contraparte de Filipinas.

\footnotetext{
${ }^{8}$ Se hace referencia a los incidentes por los límites del Mar del Sur de China y las Islas Spratly, reclamadas por China y varios países del ASEAN. En el período 1994-1996 hubo incidentes de barcos pesqueros chinos que incursionaron en áreas ocupadas por fuerzas navales de otros países. La tensión más importante se dio con Filipinas, cuando este país descubrió que China había construido instalaciones en el arrecife de Mischief, bajo control filipino.

${ }^{9}$ Las elecciones de 2014 provocaron un resultado histórico a nivel de recambio de gobierno en la India ya que fue la primera vez que el tradicional Partido del Congreso fue derrotado por el partido nacionalista hindú, Bharatiya Janata Party, el cual pudo generar gobierno por sí sólo, sin tener que recurrir a la coalición con otros partidos minoritarios.

${ }^{10}$ Dentro de los proyectos de conectividad propuestos por el gobierno de Modi se pueden destacar el proyecto trilateral de autopista que involucra a India, Myanmar y Tailandia y el ferrocarril entre Nueva Delhi y Hanoi (Mohapatra, 2014).
} 
El arribo de Narendra Modi al gobierno parece indicar que la falta de voluntad de India para actuar como potencia regional ha terminado debido a la proactividad de su administración en materia de política exterior. Los diversos viajes realizados, los acuerdos bilaterales firmados en tan corto período de gobierno y la activa participación en los foros multilaterales y en la región han respondido al objetivo de profundizar y ampliar los compromisos en materia de política exterior del país para garantizar los intereses nacionales (Sidhu y Godbole, 2015).

A pesar de esta serie de iniciativas del gobierno de Modi buscando posicionarse regionalmente, existe una fuerte crítica respecto al verdadero avance de las vinculaciones entre India y el SEA. En consecuencia, el objetivo de este trabajo es describir la forma en que la Look East Policy ha evolucionado hacia la Act East Policy y analizar las acciones llevadas a cabo por el gobierno de Modi con el fin de observar si se evidencia una acción mayor que en el pasado y una profundización en la relaciones con los países del Este de Asia. Para llevar a cabo este objetivo, se aplicó una metodología cualitativa, de sistematización de fuentes primarias y secundarias, utilizando como técnica el análisis documental y la triangulación de datos (Vieytes, 2004). Además, cabe aclarar que se tomó la decisión metodológica de estructurar el trabajo de acuerdo a tres variables de análisis, que fueron consideradas como las más pertinentes para analizar la evolución de la LEP en AEP, a saber: la institucional, la económica-comercial y la de seguridad.

\section{La dimensión institucional}

El primer paso de la LEP fue involucrar a India en el andamiaje institucional de los países del Sudeste Asiático reunidos en la ASEAN a modo de reglar las relaciones. Por tanto, la dimensión institucional de la LEP tuvo un auge muy importante en la primera fase (1994-2003) tanto con la ASEAN como a través de la iniciativa individual india. En la segunda fase (2004-2013) se observa la consolidación y ampliación de los lazos establecidos con la ASEAN, mientras que en la tercera (2014-...) el gobierno del Bharatiya Janata Party (BJP) ha propugnado ampliar los vínculos más allá del Sudeste Asiático.

Como se indicó anteriormente, la apertura hacia el Este surgió muy vinculada a la situación económica que estaba experimentando India, esto es, se intensificó el proceso de liberalización económica iniciado durante el mandato de Rajiv Gandhi ${ }^{11}$ y profundizado durante el gobierno de Rao. Sin embargo, también hubo motivos de geopolítica y de seguridad a nivel sistémico y regional que impulsaron aquel enfoque de política exterior. La redefinición de las relaciones con los países del Sudeste Asiático estuvo signada por una serie de acontecimientos que se habían constituido como amenazas a la seguridad del Estado indio y a la estabilidad regional.

En primer lugar, en 1988 se instaló un régimen militar en Myanmar bajo el liderazgo del General Ne Win. Por razones de afinidad ideológica los gobiernos de China y Pakistán intensificaron y expandieron su influencia en aquel país mientras que India, que mantenía su apoyo tradicional a las fuerzas democráticas de Myanmar, fue aislada por el nuevo ré-gimen militar. Dicha situación no era favorable para la seguridad interna de Nueva Delhi ya que podía provocar inestabilidad política en la región noreste del país.

${ }^{11}$ Durante sus cinco años de gobierno, el Primer Ministro Rajiv Gandhi visitó Indonesia, Tailandia, Myanmar Vietnam y China (Muni, 2011). 
En segundo lugar, la emergencia de China en la región se planteaba como un factor de disrupción ya que venía desplegando una batería de políticas con el fin de extender sus lazos económicos y estratégicos con los países del Sudeste Asiático.

En tercer lugar, como consecuencia del fin de la Guerra Fría, Estados Unidos había reducido oficialmente su presencia naval en el Océano Índico, razón que condujo al gobierno indio a ampliar su programa naval para poder defender sus propios intereses en aquellas aguas. Dicha situación llevó a acrecentar las suspicacias de los países de la región respecto del accionar de Nueva Delhi, por lo cual se tomó la decisión de gobierno de buscar alternativas de política exterior para acallar tales rumores.

Por último, ante el conflicto interno que ocurría en Camboya ${ }^{12}$, los miembros de la ASEAN exhortaron a India a tener un mayor compromiso con la región, justamente para poder dar solución a la cuestión así como para balancear la creciente presencia de China en el Asia-Pacífico (Muni, 2011).

En la fase inicial de la LEP el gobierno indio intensificó sus relaciones bilaterales con los miembros de la ASEAN adoptando dos formas de acercamiento según su distinción entre miembros fundadores (Indonesia, Malasia, Las Filipinas, Singapur y Tailandia) y miembros nuevos (Myanmar, Laos, Camboya y Vietnam) (Jyoti, 2013 y Muni, 2011). Entre los miembros fundadores de la ASEAN, Singapur jugó un rol fundamental como facilitador de los canales institucionales que se establecieron con el grupo regional (Muni, 2011), por lo cual no ha sido una casualidad que la LEP haya sido lanzada en aquel país del Sudeste Asiático.

En cuanto a los miembros nuevos, el caso de Myanmar merece especial atención ya que las relaciones con este país representaron un giro en la política india a mediados de los 90', desde una postura principista (característica de su política exterior durante la Guerra Fría) a una de corte más pragmática ${ }^{13}$. El gobierno indio entendía que era preferible el diálogo más que el aislamiento, para favorecer una apertura democrática, posición que era compartida por la mayoría de los miembros de la ASEAN (Mato Bouzas, 2009). Entre los motivos que incidieron sobre Nueva Delhi para tal 'vuelco diplomático' se deben considerar que Myanmar es un Estado fronterizo clave como territorio de tránsito hacia los países del Sudeste Asiático (hecho fundamental para aumentar su comercio) y que Nueva Delhi tenía interés en las fuentes de abastecimiento energético de este país.

Se puede observar entonces que la intensificación de algunos lazos bilaterales allanaron el camino para una mayor actuación dentro del proceso regional ASEAN. Las sucesivas promociones en la categoría de socio de la Asociación -tal como se dio a conocer en la introducción, en 1992 India se convirtió en Socio de Diálogo Sectorial; luego en 1995 fue elevado a Socio de Diálogo Pleno, extendiendo las áreas de cooperación- le permitió a India acceder a la membresía en otros foros regionales iniciados en el seno de la Aso-

${ }^{12}$ La crisis humanitaria de Camboya consistió en una serie de eventos que se extendieron desde 1969 hasta 1991 en donde miles de camboyanos murieron o fueron desplazados por la situación de conflicto interno y guerra de guerrillas que se produjo con posterioridad. Es preciso analizar esta crisis como un conflicto enmarcado en la lógica de la Guerra Fría.

${ }^{13}$ Este cambio provocó numerosas críticas tanto dentro como fuera de India. Sin embargo, importa adelantar que en noviembre de 2015 se llevaron a cabo elecciones libres en Myanmar siguiendo el proceso iniciado en 2011, cuando un gobierno civil fue establecido en el poder después de más de cincuenta años de gobierno militar (BBC, 2015). 
ciación, como el Foro Regional ASEAN (ARF) y la Cumbre del Este de Asia (más conocido por su acepción inglesa 'East Asia Summit').

Por tanto, el hecho de que India haya sido ascendida a la categoría de Socio de Diálogo Pleno en 1995 le dio el acceso al ARF, que había sido creado en el 270 Encuentro Ministerial ASEAN de $1993^{14}$. Dicho mecanismo funciona como un foro de seguridad regional, siendo su objetivo central promover el diálogo y la consulta así como la construcción de confianza gracias a la diplomacia preventiva.

El caso de la participación de India en la primera Cumbre del Este de Asia, como otro foro de diálogo que surgió a partir de la ASEAN, fue cuestionado por China y por Malasia argumentando que aquel país "no pertenecía al Este". Esto se debe a que el mecanismo regional fue recomendado por el ASEAN+3 (China, Japón y Corea del Sur) ${ }^{15}$ en 2002 como de pertenencia exclusiva para estos países. Sin embargo, fueron los propios miembros de la ASEAN reunidos en una conferencia ministerial en 2005 que decidieron abrir la participación a Australia, India y Nueva Zelanda ${ }^{16}$. Por otra parte, vale aclarar que fue Singapur quien ayudó a sortear la oposición de China y Malasia a la participación de Nueva Delhi en la primera cumbre.

De lo detallado se puede analizar que hubo una doble oposición a la participación de India en la Cumbre del Este de Asia. En primer lugar, por la forma restringida en que fue concebido tal foro de diálogo limitando los miembros extra-ASEAN a China, Japón y Corea del Sur y, en segundo lugar, una vez conformada la Cumbre, China y Malasia dieron a conocer su rechazo de manera expresa. Esta negación puede relacionarse con el avance que el gobierno indio había conseguido en 2003 con la firma del Acuerdo Marco de Cooperación Económica con los países de la ASEAN, que sentaba las bases para lograr un Acuerdo de Libre Comercio en el futuro, que podría menoscabar sus intereses.

Ahora bien, la integración institucional de India con los países del Sudeste Asiático no quedó confinada solamente a las relaciones instauradas con la ASEAN. Durante la primera fase de la LEP, Nueva Delhi promovió el establecimiento -o su participación- de nuevas iniciativas regionales y sub-regionales como la Asociación Regional para la Cooperaciónde los Países Ribereños del Océano Índico (IORARC, por su siglas en inglés) que data de $1997^{17}$; la Iniciativa de la Bahía de Bengala para la Cooperación Multisectorial científica, técnica y económica ${ }^{18}$ (BIMSTEC, por sus siglas en inglés) fundada en 1997, con el objetivo de pro-

${ }^{14}$ El ARF es una plataforma de diálogo multilateral en la región Asia-Pacífico conformado por los diez miembros de la ASEAN más los Socios de Diálogo de la Asociación, es decir: Australia, Canadá, China, India, Japón, Nueva Zelanda, Rusia, Corea del Sur, los Estados Unidos y la Unión Europea

${ }^{15}$ Así se le denomina al Tratado de Libre Comercio que involucra a los países mencionados y que está vigente desde 1997.

${ }^{16}$ En 2011 se sumaron Estados Unidos y la Federación Rusa.

${ }^{17}$ Es una plataforma para la cooperación económica y la cooperación técnica regional. En la actualidad reúne a diecinueve países: Australia, Bangladesh, India, Indonesia, Irán, Kenia, Malaysia, Madagascar, Mauritania, Mozambique, Omán, Seychelles, Singapur, Sudáfrica, Sri Lanka, Tanzania, Tailandia, Emiratos Árabes Unidos y Yemen. Asimismo posee cinco socios de diálogo: China, Egipto, Francia, Japón, Gran Bretaña y tres observadores, Indian Ocean Research Group (IORG), Indian Ocean Tourism Organisation (IOTO) y Omán. Información extraída de http://www.iora.net/ [Consultado el día 13-05-2017].

${ }^{18}$ Los países miembros de la Iniciativa de Bengala son Myanmar, Bangladés, India, Sri Lanka, Tailandia, Bután y Nepal. Información extraía de http://www.bimstec.org/ [Consultado el día 14-05-2017]. 
mover la cooperación económica, en inversiones, turismo, zonas de pesca, agricultura, entre otros; o la Cooperación Mekong-Ganges (MGC) ${ }^{19}$ establecida en 2000 para ampliar las relaciones económicas y sociales. En consecuencia, siguiendo a Naidu (2013: 65) "[a] distinct feature of India's political engagement with East Asia is that, as with ASEAN, a variety of institutional arrangements have been created to ensure that interactions constantly take place at various levels and relations get strengthened continuously" ${ }^{\prime 20}$.

La segunda fase de la LEP, que coincidió con el gobierno de Manmohan Singh (2003-2014), se dedicó a llenar de contenido las instancias regionales inauguradas durante la década anterior, aumentando los montos de cooperación para cada organización. A su vez, se concentraron esfuerzos en profundizar el nivel económico-comercial con la ASEAN, como se verá en el apartado siguiente. Durante ese período se practicó un mayor activismo en ámbitos como IBSA y BRICS, lo cual no implicó que disminuyera la relevancia dada a los países del Este de Asia en la década anterior.

Durante la tercera fase, renombrada Act East Policy por el Primer Ministro Narendra Modi, dicha política ha mostrado signos que permiten proyectar algunos cambios respecto del gobierno anterior. En este sentido, una de las señales que ha dado su gobierno es la voluntad involucrarse en todas las propuestas chinas para Asia-Pacífico. Ejemplo de ello fue la visita oficial que realizó Modi en mayo de 2015 a China marcada por una clara impronta económica -dejando por fuera las cuestiones de seguridad-, movimiento que puede ser interpretado a la luz de las intenciones del gobierno indio de formar parte de la Organización de Cooperación de Shangai (OCS) como miembro pleno así como del Foro de Cooperación Económica Asia-Pacífico (más conocido como APEC) ${ }^{21}$. Asimismo lo demuestra la participación de India como miembro fundador del Banco Asiático de Inversiones e Infraestructura -de iniciativa China- y el Nuevo Banco de Desarrollo BRICS, en donde China tiene una participación mayoritaria.

Aquellos ajustes pueden estar mostrando, por un lado, que India no pretende desaprovechar las oportunidades que pueden procurarle un mayor crecimiento económico, y por otro, que está actuando estratégicamente para calmar al 'dragón chino' debido a la mayor asertividad que su gobierno viene aplicando en las relaciones con los países de Asia-Pacífico. Al respecto, en 2014 India anunció el lanzamiento del Banco de Desarrollo de la Asociación Surasiática para la Cooperación Regional (SAARC) ${ }^{22}$ (Roychoudhury et all, 2015). Este último, es un claro intento de emular la iniciativa china con el establecimiento

${ }^{19}$ El Grupo de Cooperación Mekong-Ganges (MGC) está constituido por la India y cinco estados miembros de la Asociación de Naciones del Sudeste Asiático lindantes con esos ríos -Camboya, Laos, Myanmar, Tailandia y Vietnam.

20 "Una característica distintiva activismo político de India con el Este de Asia es que, tal como con la ASEAN, una gran variedad de acuerdos institucionales han sido creados para asegurar que las interacciones tengan lugar en varios niveles y que las relaciones se fortalezcan constantemente" (Traducción por parte de las autoras).

${ }^{21}$ En julio de 2015, el Consejo de Jefes de Estado de la OCS admitió a India y Pakistán como miembros plenos a partir del año 2016. Mientras que las negociaciones en la APEC continúan demoradas respecto de la incorporación de nuevos miembros (The Hindu, 11/07/2015).

${ }^{22}$ Sin embargo, ya se están escuchando voces contrarias en el gobierno de Modi respecto de la inviabilidad de la propuesta, teniendo presente que India debería ser el mayor socio aportante (Bhutan News Network, 21/08/2015). 
del Banco Asiático y al mismo tiempo, contrarrestar la estructura de incentivos que China supo extender a nivel regional, atentando contra los intereses indios (Sahni, 2013).

En las páginas siguientes se particularizará sobre los frutos cosechados por India gracias al avance a nivel institucional tanto con la ASEAN como por iniciativa individual en términos económicos y de seguridad.

\section{La dimensión económica-comercial}

El diseño de la LEP tenía como eje central ayudar al crecimiento económico del país. En la primera etapa de su ejecución se observan los primeros resultados: entre 1993 y 2003 , el comercio creció a un promedio anual del 12\%, pasando de US\$ 2 mil millones a US\$ 12 mil millones (Ministerio de Asuntos Exteriores de India, 2017).

Como corolario del incremento de los intercambios políticos, diplomáticos y económicos, que resultaron luego de esta primera etapa, se comenzó a poner énfasis en el objetivo de alcanzar un Acuerdo de Libre Comercio (ALC) para continuar profundizando el proceso de integración entre ambas regiones. Por el lado de India, los motivos fundamentales fueron la necesidad de diversificar su comercio con la ASEAN -tanto en término de productos como de destinos- así como no quedar relegada tras las negociaciones que China había entablado con dicho bloque para alcanzar un ALC. Por su parte, los países pertenecientes a la ASEAN, luego de atravesar la crisis económica de 1997, comenzaron a considerar al mercado indio como un destino con un enorme potencial para la colocación de sus productos y que a la vez le permitiera disminuir su dependencia de sus socios comerciales tradicionales (Yahya, 2003).

De este modo se iniciaron las negociaciones en la Primera Cumbre India-ASEAN en Nueva Delhi en el año 2002, en donde se comenzó a definir la cooperación en materia de comercio exterior. Esta situación desembocó en la segunda Cumbre India-ASEAN en el año 2003 en Bali, donde se suscribió el Acuerdo Marco en Cooperación Económica (FACEP, por sus siglas en inglés), que preveía alcanzar un ALC que abarcara bienes, servicios e inversiones y establecía dos fechas de comienzo, teniendo en cuenta los diferentes niveles de desarrollo económico de los miembros: en primera instancia el 31 de diciembre de 2011 rigió para Brunei, Indonesia, Malasia, Singapur, Tailandia e India, mientras que el 31 de diciembre de 2016 era la fecha indicada para Camboya, Laos, Myanmar, Vietnam y Filipinas.

Tras seis años de intercambio de diferentes opiniones y posiciones respecto al acuerdo, el 13 de agosto de 2009 se firmó en Bangkok el ASEAN-India Trade-in-Goods Agreement. El potencial de este Acuerdo de Comercialización de Bienes quedó al descubierto en los primeros años de ejecución. Mientras que en el período 2007-2008 el intercambio de mercancías entre ASEAN-India promediaba en los U\$S 40 mil millones, durante el período 2014-2015 el comercio total entre estos mercados rondó los U\$S 76 mil millones. Sin embargo, para el ciclo 2015-2016 el intercambio comercial disminuyó a US\$ 65 mil millones debido a la caída de los precios internacionales de los commodities, la caída de la demanda de los países del SEA y la desaceleración de la economía mundial. El dato económico negativo para la India ha sido la persistencia del signo deficitario en su balanza comercial con la ASEAN (Ministerio de Asuntos Exteriores de India, 2017) ${ }^{23}$.

${ }^{23}$ Respecto a los productos intercambiados, las exportaciones de India se centran en combustibles minerales (fuel oil); perlas y piedras preciosas y semipreciosas; carnes; químicos orgánicos; barcos, botes y 
A pesar del déficit en la balanza de pagos con la ASEAN, la conclusión de las negociaciones sobre el Acuerdo de Comercialización de Servicios e Inversiones el 20 de diciembre de 2012 fue un dato alentador para la economía india. Dada su alta competitividad en la exportación de servicios y la disponibilidad de mano de obra calificada, se observaron im-portantes oportunidades en economías tales como Malasia, Singapur y Tailandia.

Respecto a las inversiones, que complementan la profundización del vínculo económico, ASEAN representa un $12,5 \%$ de las inversiones extranjeras en India. El flujo de las inversiones desde ASEAN, entre principios de 2000 y mayo de 2016 fue de US\$ 49 mil millones, mientras que las inversiones indias en los países miembros de ASEAN, desde principios de 2007 hasta marzo de 2015, fueron aproximadamente de US\$ 38 mil millones (Ministerio de Asuntos Exteriores de India, 2017). Estas cifras muestran que las inversiones están por debajo de su potencial, y el Primer Ministro Modi ha marcado la intención de ayudar a las empresas indias a aumentar la inversión extranjera directa en la región (Parameswaran, 2014).

El sector privado es, para ambas regiones, un actor importante en las vinculaciones externas, y por lo tanto han trabajado para lograr un mayor involucramiento del mismo. En este orden, se creó en 2003 el Consejo de Negocios ASEAN-India, el cual se estableció como un foro que sirve de plataforma de networking, donde los actores del sector privado pueden conectarse y compartir ideas. Este Consejo ha organizado el primer cónclave del sector en julio de 2016 (Ministerio de Asuntos Exteriores de India, 2017)

Con la victoria de Narendra Modi en las elecciones de 2014 se dió el momento propicio para posicionar a India estratégicamente en el Asia-Pacífico (Rajendram, 2014), a través de un nuevo plan de acción con énfasis en la profundización de las conexiones en materia comercial y de circulación de personas. Así, una de las primeras acciones fue la firma del Acuerdo de Libre Comercio en Servicios e Inversiones, que tuvo lugar en septiembre de 2014 y entró en vigor el $1^{\circ}$ de julio de 2015, completándose -junto al ALC de Bienes- la Sociedad Económica Comprehensiva (Babu, 2013) ${ }^{24}$.

Durante la participación del Primer Ministro Modi en la Cumbre India-ASEAN celebrada en noviembre de 2014, se enfatizó en la necesidad de aumentar los intercambios comerciales y profundizar de manera general, los lazos económicos. Particularmente, referenció la rapidez con la que deseaba se concretasen proyectos en materia de infraestructura, comercio de manufacturas, agricultura, desarrollo y renovación urbana de ciudades (Parameswaran, 2014).

Dentro de este marco, el gobierno anunció dos importantes iniciativas. En primer lugar, la decisión de crear una empresa de desarrollo de proyectos (con un capital inicial de US\$ 100 mil millones), con el objetivo de establecer centros de producción manufacturera

estructuras flotantes; entre otros. Por su parte, importa de los países de la ASEAN, combustibles minerales; grasas y aceites vegetales y animales; maquinaria y equipos eléctricos; químicos orgánicos; productos plásticos; madera y productos de madera; entre otros. De lo descripto se desprende que predomina la exportación india de productos manufacturados y la importación de bienes intermedios desde los países del Sudeste Asiático (Ministerio de Comercio e Industria de India, 2016).

${ }^{24}$ Cabe recalcar que también se ejecutará de manera diferenciada de acuerdo al estado de situación económica de sus miembros. Es decir, que habrá un esquema de compromisos específico para Filipinas, otro para Indonesia y un tercer esquema para el resto de los estados miembros de la ASEAN. 
en Camboya, Laos, Myanmar y Vietnam; y segundo, la creación de un fondo de US\$ 1 mil millones para aumentar la conectividad entre India y los estados de la ASEAN (Bhatia, 2016). En este sentido, los principales proyectos son: la Autopista Trilateral India-MyanmarTailandia, el ferrocarril entre Nueva Delhi y Hanoi y el Proyecto Multimodal de Kaladan.

India ha realizado importantes progresos en el desarrollo de la Autopista Trilateral y en el proyecto Multimodal de Kaladan. Se ha alcanzado el consenso respecto al Acuerdo Automotor India-Myanmar-Tailandia, el cual permitirá lograr el movimiento de vehículos de pasajeros, particulares o de carga en los caminos que unen los tres países. También se encuentra bajo consideración la extensión de la autopista a Camboya, Laos y Vietnam. Sin embargo, el incremento de la conectividad marítima y aérea entre ASEAN e India, y la transformación de dichas vías en corredores económicos se encuentra aún bajo discusión entre las partes (Ministerio de Asuntos Exteriores de India, 2017).

Por otro lado, India ha provisto de asistencia financiera a los países del ASEAN para llevar a cabo distintos tipos de actividades de cooperación, a través de tres fondos: a) Fondo de Cooperación ASEAN-India: creado en 2009, ha contribuido con US\$ 50 millones para la implementación de los Planes de Acción en sectores como el político, el económico y el socio-cultural, y se ha propuesto, en 2016, aumentar el aporte a otros US\$ 50 millones; b) Fondo para el Desarrollo de la Ciencia y la Tecnología ASEAN-India: creado en 2007, ha contribuido con US\$ 1 millón para promover la colaboración conjunta en proyectos de investigación en ciencia y tecnología, y el gobierno ha anunciado un incremento del fondo a US\$ 5 millones para el período 2016-17; y c) Fondo Verde ASEAN-India: creado en 2007, ha participado con una contribución inicial de US\$ 5 millones, para actividades vinculadas con el medio ambiente y el cambio climático (Ministerio de Asuntos Exteriores de India, 2017).

Narendra Modi ha hecho especial hincapié en el tamaño de la economía india y de la de aquellos estados del SEA en su conjunto, y es por ello que comprende que un aumento en los lazos económicos y comerciales entre India y la región supondría la adquisición de mayor poder a nivel internacional. Asimismo, podría balancear el poder chino en esta parte del mundo: "Tenemos la fuerza y el potencial de una población joven. En India, 800 millones de personas debajo de los 35 años constituyen una gran oportunidad" (Modi, 2014).

No obstante, para el año 2013 comenzó a sentirse la desaceleración del comercio y de la economía mundial, y el crecimiento de India sufrió una fuerte caída. Esta situación generó en el nuevo gobierno la necesidad de desarrollar un plan, parte de una iniciativa más amplia de construcción nacional, para transformar a India en un centro global de diseño y de manufacturas. "Make in India" fue la respuesta del Primer Ministro Modi a esta situación. El programa, lanzado en septiembre de 2014, identificó varios desafíos que enfrenta India y que a través de reformas y actividades de promoción del país pueden mejorarse, a saber: mantener un crecimiento de alrededor del 10\% por tres décadas; facilitar los procedimientos para hacer negocios; hacer de India un eslabón en las cadenas globales de valor; estimular el sector comercial doméstico; transformar a India en un país con una economía basada en las manufacturas; capacitar a la mano de obra joven; hacer de India un centro de investigación y desarrollo; encarar un proceso de urbanización junto al desarrollo de infraestructura (Make in India, 2017).

En apenas dos años, las importantes reformas que se han llevado a cabo ponen de manifiesto que India se ha convertido en el país con el mayor crecimiento económico del mundo, con un promedio del 7\% para el período 2016-2017. El sector manufacturero es el 
que más se ha desarrollado, encontrándose actualmente en la sexta posición a nivel mundial. También ha comenzado el camino a convertirse en un centro de manufacturas de alta tecnología, ya que empresas como Siemens, HTC, Toshiba y Boeing están instalándose o en el proceso de hacerlo (Make in India, 2017). Estos pocos indicadores demuestran que el gobierno indio ha pasado a la acción en la búsqueda del desarrollo del país.

Independientemente del hecho de que la desaceleración de la economía y del comercio mundial han impactado en las relaciones entre India y ASEAN, se observa que desde 2003 se ha dado un salto cuantitativo y cualitativo en las relaciones económico-comerciales entre ambas regiones. Además, desde 2014, se ha ampliado el horizonte comercial a otros países del Este de Asia, como Japón y Australia.

\section{La dimensión de seguridad y defensa}

En lo que respecta al accionar de India en materia de seguridad, debemos considerar que comenzó a tener un rol más destacado en los mecanismos de cooperación de la región a partir de la segunda fase de la $\operatorname{LEP}^{25}$. Por un lado, y de acuerdo a las declaraciones del Ministro de Relaciones Exteriores, Yashwant Sinha durante la administración de Manmohan Singh, esto se debe a que:

"The first phase of India's 'Look East' policy was ASEAN-centred and focussed primarily on trade and investment linkages. The new phase of this policy is characterised by an expanded definition of 'East', extending from Australia to East Asia, with ASEAN at its core. The new phase also marks a shift from trade to wider economic and security issues, including joint efforts to protect the sea-lanes and coordinate counter-terrorism activities" (Discurso del Ministro de Relaciones Exteriores Shri Yashwant Sinha, 29 de septiembre de 2003).

Por otro lado, los ensayos nucleares llevados a cabo por el gobierno de Atal Bihari Vajpayee en el año 1998 (conocido como Pokhran-II), que en los días posteriores fueron replicados por las pruebas nucleares de Pakistán, produjeron gran consternación a nivel mundial y regional. A pesar de que la decisión del gobierno indio podría haber perjudicado el proceso de integración con los países del Sudeste Asiático -recordemos que se había constituido como Socio de Diálogo Pleno de la ASEAN en 1995, estatus que le permitió formar parte del ARF en 1996-, la Reunión de Ministros de Relaciones Exteriores de la ASEAN de julio de 1998 se limitó a emitir un comunicado conjunto deplorando las demostraciones nucleares y el ARF sólo instó a todos los Estados a adherirse al Tratado de No Proliferación Nuclear. Esto se debe a que mientras algunos Socios de Diálogo de la

\footnotetext{
${ }^{25}$ Sin embargo, a propuesta de la marina india se creó un foro de fuerzas navales regionales en 1995 que se reuniera bianualmente con el objetivo de generar un intercambio asiduo para incrementar la cooperación marítima. La primera reunión dio lugar a una serie de ejercicios navales conjuntos (conocidos como MILAN) con varios países del Sudeste Asiático para promover la operatividad conjunta y, de esa manera, generar un mayor grado de confianza. Los países participantes compartían el deseo de querer balancear la creciente influencia de China en Asia-Pacífico mientras que India pretendía posicionarse como líder regional al realizar tal demostración de fuerza. Asimismo, Nueva Delhi intentaba detener el tráfico de armas a través de la Bahía de Bengala, armamento que se dirigía a los insurgentes del noroeste de su propio territorio (Jyoti, 2013).
} 
ASEAN buscaban condenar a Nueva Delhi, el punto de vista mayoritario al interior de la ASEAN supuso que el ARF no debía ser utilizado para denunciar a sus participantes.

Lo expuesto debe ser analizado contemplando que el accionar de India fue repudiado enérgicamente a nivel individual por varios de los miembros del ARF. A saber, Filipinas y Tailandia tomaron las posturas más duras dentro de la ASEAN. Estados Unidos y Japón impusieron sanciones económicas además de la expresa desaprobación públi$\mathrm{ca}^{26}$; Rusia, China, Australia y la Unión Europea se limitaron a reprobar los ensayos y Nueva Zelanda retiró a su funcionario de Embajada en Nueva Delhi (Singh, 1999).

La benevolencia de la ASEAN respecto a la reafirmación de la política nuclear de India merece ser comparada con la postura tomada por China, debido a que la estrategia diplomática empleada por el gobierno indio para hacer frente al rechazo internacional fue justificar las pruebas nucleares en 'la amenaza china'. El círculo de colaboradores más cercanos a Vajpayee, entre ellos el ministro de Defensa George Fernandes, argumentaron que los ensayos nucleares habían sido una respuesta ante una potencial amenaza proveniente de su vecino chino, quien a su vez mantenía una "entente" con Pakistán (González Castañeda, 2010: 107).

Ante tales declaraciones, China sólo se restringió a una protesta diplomática y cuando tuvo la oportunidad de sentar posición en el conflicto armado de Kargil de 1999 que enfrentó a India y Pakistán ${ }^{27}$, se mantuvo neutral. Por otra parte, ese mismo año se reanudaron las actividades de la Comisión Conjunta de Trabajo entre India y China, gracias a la iniciativa china, que habían sido suspendidas debido a las pruebas nucleares ${ }^{28}$. En tanto, acordamos con Mario González Castañeda cuando afirma que:

“(...) teniendo en cuenta el proceso de reconciliación emprendido entre los dos países a inicios de la década de los noventa, era riesgoso (para India) emplear un argumento de tal magnitud. Ahora bien, los ensayos nucleares llevaron a un fuerte y rápido cambio de imagen de India en la dirigencia china y en la población en general (...) gracias al éxito de las reformas de liberalización económica, la difusión de las películas de Bollywood, el triunfo de las participantes indias en los concursos internacionales de belleza y, obviamente, del desarrollo de su programa nuclear" (Gonzalez Castañeda, 2010: 112).

El escenario cambió a partir de los atentados terroristas al World Trade Center y al Pentágono en Estados Unidos. El 11-S pasaría a securitizar la agenda a nivel internacional, y condujo a un cambio de rumbo en la estrategia norteamericana en el Sur de Asia. En este

${ }^{26}$ Estados Unidos suspendió la ayuda oficial al desarrollo (excepto la ayuda humanitaria) y las líneas de crédito otorgadas al país y prohibió algunas exportaciones de tecnología y de defensa (CNN, 13/05/1998). Por su parte, Japón congeló el otorgamiento de un préstamo por mil millones de dólares que estaba por entregar.

${ }^{27}$ Se trató de un conflicto armado entre India y Pakistán en el Kargil que se emplaza en la región de Cachemira. Siguiendo a Frazier (2000: 12) "the fact that China gave no overt support for Pakistan was perceived by India as a significant change in China's stance on the Kashmir issue.

${ }^{28}$ La Grupo de Trabajo Conjunto es un mecanismo bilateral que tiene como fin reunirse regularmente para discutir la disputa sobre la frontera y crear medidas de construcción de confianza entre ambos estados. El primer Grupo de Trabajo Conjunto fue acordado en 1989 durante la visita del Primer Ministro indio Rajiv Gandhi a China (Frazier, 2000). 
sentido, es representativo que la Administración de George W. Bush haya levantado las sanciones económicas impuestas a Nueva Delhi durante la era Clinton, el 22 de septiembre de $2001^{29}$.

De este modo, India pasó a ser 'mejor entendida' tanto desde el punto de vista de Estados Unidos como de sus propios vecinos regionales respecto de los conflictos transfronterizos que la afectaban, que pasaron a ser considerados como parte de aquel nuevo fenómeno definido como terrorismo internacional. Bajo este marco se propusieron nuevos mecanismos multilaterales y bilaterales de seguridad regional tanto por iniciativa de India, de los países del Sudeste Asiático reunidos en la ASEAN o por otras potencias regionales.

En cuanto a los mecanismos intra-ASEAN que contaron con la participación de Nueva Delhi es dable mencionar la firma de la Declaración Conjunta para el Combate del Terrorismo India-ASEAN en octubre de 2003 (Muni, 2011). Además durante la $10^{\circ} \mathrm{Cum}$ bre India-ASEAN de 2012 se relanzó la "Asociación India-ASEAN para la paz, el progreso y la prosperidad, la estabilidad y el desarrollo compartido", que había sido anunciada en noviembre de 2004. Para tal fin se preparó un plan de acción que tiene como objetivo fortalecer la cooperación política, económica y de seguridad así como en las siguientes áreas: seguridad marítima, seguridad ambiental, energía y antiterrorismo ${ }^{30}$.

Asimismo, la Asociación estableció las Reuniones de Ministros de Defensa ASEAN (ADMM, por sus siglas en inglés) en 2006 con el fin de cooperar en casos de desastre y crisis humanitaria. Importa enfatizar que la cuarta reunión ADMM en 2010 fue renombrada como $\mathrm{ADMM}+8$ al incluir ocho nuevos miembros que tienen la categoría de ser "Socios de Diálogo"31, entre los que se encuentra India.

En lo que respecta a los mecanismos extra-ASEAN, India se sumó a la iniciativa japonesa que derivó en el Acuerdo de Cooperación en el Combate a la Piratería y Robo Armado contra Buques en Asia (ReCAAP, por sus siglas en inglés) firmado en el año 2004, por el cual se coopera para asegurar las aguas regionales. En un principio el acuerdo involucró a dieciséis países del Este de Asia aunque pronto se expandió a otros países extraregionales ${ }^{32}$.

En lo que respecta al nivel bilateral, es preciso particularizar en la dinámica de seguridad de India con China, Japón y Estados Unidos debido al peso específico que tienen cada uno de estos estados en el Este y Sur de Asia. Cabe recordar que las actuales relaciones entre India y China han estado permeadas por los resultados de la guerra de 1962, es decir, por las disputas fronterizas irresueltas cimentando la desconfianza mutua. Aún antes de lanzarse la LEP los dos países iniciaron una serie de intercambios de alto nivel en 1989 que buscaban construir mecanismos de confianza mutua y que tuvieron como consecuencia la disminución de las tropas de frontera y el intercambio de notificaciones de ejercicios militares.

${ }^{29}$ El gobierno de Japón se acopló en la misma dirección, es decir, quitó las sanciones impuestas en 1998.

${ }^{30}$ Información extraía de la página web http://www.asean.org/news/item/overview-of-asean-indiadialogue-relations [Consultado el día 05-04-2016].

${ }^{31}$ Los ocho miembros son: Japón, Corea del Sur, República Popular China, Australia, Nueva Zelanda, Rusia y Estados Unidos.

${ }^{32}$ Los países signatarios del acuerdo son: Brunei, Camboya, Myanmar, Laos, Las Filipinas, Singapur, Tailandia, Vietnam, Bangladesh, China, India, Japón, Corea del Sur, Sri Lanka, Estados Unidos, el Reino Unido, Dinamarca, Noruega, Nueva Zelanda y Australia. Información extraída de la página web Información extraída de la página web http://www.recaap.org/ [Consultado el día 05-04-2016]. 
No obstante, hubo dos acontecimientos que estancaron esos avances. El primero de ellos se refiere al aumento de exportaciones de armas de Beijing a Myanmar durante los años 90' y con ello, la preocupación del gobierno indio de que los grupos insurgentes del noreste de su país recibieran apoyo de China a través del territorio birmano y que la influencia de Beijing en su vecino meridional iba dirigida a obtener una mayor presencia en el Índico, lo que sin duda afectaba a la seguridad y ambiciones de la India en la región (Mato Bouzas, 2007). El segundo hecho fueron las ya mencionadas detonaciones nucleares que realizó India en 1998. Con posterioridad al Pokhran II, el deshielo en las vinculaciones entre China e India se produjo principalmente a causa del incremento de la interdependencia económica. En el año 2008 China se convirtió en el principal socio comercial de India, así como las compañías chinas pasaron a ocupar los primeros puestos en inversiones en el país vecino.

Sin embargo, las relaciones entre China e India están caracterizadas por la tensión constante entre la cooperación y la competencia. En lo que respecta a la cooperación, y tal como hemos puntualizado a lo largo del trabajo, ambos países forman parte de una serie de organismos regionales en temáticas de comercio, seguridad y defensa en donde trabajan procurando el beneficio mutuo. No obstante, ambos países compiten debido a que desean extender su influencia política en la región así como hacerse con el control de los recursos energéticos, las materias primas y los mercados que necesitan para impulsar sus economías en crecimiento.

Ante los vaivenes en los vínculos con China, la construcción de seguridad en el Este de Asia no puede considerarse de forma acabada desatendiendo la presencia de Estados Unidos y Japón. Como se ha dado a conocer, iniciado el siglo XXI la administración George W. Bush cambió su percepción en cuanto al rol del 'elefante asiático', pasando a considerarlo una pieza clave como pivot de la cruzada antiterrorista en el Sur de Asia. De esta manera la mejora de las relaciones entre India y Estados Unidos tuvieron su punto cúlmine con la firma del tratado nuclear en $2008^{33}$ que condujo a transparentar el programa nuclear indio.

India entonces se elevó al estatus de aliado estratégico para Estados Unidos. Ambos países incrementaron los ejercicios militares conjuntos y los intercambios de delegaciones comerciales y Washington amplió el acceso a sus mercados (principalmente dentro de la industria del software). Tal es así que comenzó a ser concebida dentro de los círculos de gobierno norteamericano como un gran poder emergente del nuevo siglo (Giridharadas, 2009). Dentro de este cambio de percepción en la política exterior del país del Norte debemos tener muy presente que para la administración de George W. Bush contener la influencia de China en la región era un factor crucial.

En cuanto a las relaciones entre India y Japón, desde que el gobierno chino ha comenzado a desplegar una política exterior más asertiva en el Sudeste Asiático (principalmente en lo que se refiere a los reclamos territoriales de ultramar en el Mar del Sur de

${ }^{33}$ Ambos países concluyeron un acuerdo para el desarrollo de energía nuclear con fines civiles y pacíficos en octubre de 2008. Con dicho tratado, el gobierno de India abrió sus puertas a la vigilancia de 14 de sus 22 instalaciones nucleares a la Organización Internacional de la Energía Atómica (OIEA), organismo encargado de controlar el uso responsable de la energía nuclear. Por su parte, Estados Unidos se comprometió a compartir tecnología y material nuclear con este país asiático que no suscribió el Tratado de No Proliferación (TNP) (Dussort, 2013). 
China), la cooperación en el área de seguridad se ha vuelto la más comprehensiva en la historia de los dos países. Es ilustrativo el hecho de que Tokio y Beijing han establecido una "asociación estratégica y global" desde el año 2006, avanzando significativamente en materia de defensa conjunta.

En definitiva, avanzada la segunda década del siglo XXI, Manmohan Singh dio paso al nuevo gobierno del BJP habiendo logrado concretar mecanismos de cooperación en materia de defensa con todos los países del Este de Asia, excepto Corea del Norte (Naidu, 2013). En ese sentido, firmó acuerdos con algunos países miembros de la ASEAN como Vietnam, Malasia, Laos y Singapur para abastecerlos de equipamiento de defensa, ayudar a actualizar y reparar sus sistemas armamentísticos y ofrecerles entrenamiento a su personal militar (Muni, 2011).

Por último, después de dos años y medio de lanzada la AEP, se puede observar que el gobierno de Narendra Modi ha avanzado sustancialmente respecto de las cuestiones relativas a la seguridad con sus vecinos del Este. Al mismo tiempo cabe puntualizar que el BJP inauguró su gestión teniendo como eje rector la economía y justamente las cuestiones de defensa y seguridad no quedaron ajenas a ello. En consecuencia, la campaña "Make in India" desplegada por el gobierno para fortalecer la producción nacional e incrementar las exportaciones del país también ha tenido su correlato en la industria armamentística, siendo puesta a prueba con los países del Sudeste Asiático.

A modo de ejemplo, el gobierno de Modi ha reforzado la cooperación en materia de defensa con Singapur, Malasia y Tailandia. Durante el año 2015, el Primer Ministro realizó visitas de Estado a Singapur y Malasia que fueron precedidas por una gira del Jefe Naval indio por Singapur y Tailandia. Durante la visita del Primer Ministro indio a Malasia se firmó una declaración conjunta para la cooperación de las guardias costeras de ambos países y para establecer vínculos entre los dos centros nacionales para el mantenimiento de la paz (Centre for UN Peacekeeping of India y Malaysian Peacekeeping Centre). En cuanto a los acuerdos firmados con Singapur, Modi logró la firma de un Memorándum de Entendimiento sobre seguridad cibernética, aviación civil, cooperación en el campo de la planificación, cooperación en el combate contra el tráfico ilícito de narcóticos y sustancias psicotrópicas, entre otros (Ranjan, 2016).

En cuanto a su actuación dentro de los foros de diálogo multilateral, el Primer Ministro Modi se refirió a la disputa por la soberanía sobre las islas del Mar de China Meridional en la décima reunión de la East Asia Summit al manifestar que su país "shares with ASEAN a commitment to freedom of navigation, over flight and unimpeded commerce, in accordance with accepted principles of international law, including the 1982 UN Convention on the Law of the Sea" (Modi, 2015). Si bien sus palabras carecen de contundencia, puede vislumbrarse cierto compromiso con los países del SEA en sus reclamos hacia China, especialmente porque más del $40 \%$ del comercio indio surca aquellas aguas.

En resumen, ya desde la segunda fase de la LEP en adelante India ha demostrado un deseo de cumplir un rol mayor en Asia en la construcción de la seguridad regional. Esta clara posición ha llevado a que los países de la ASEAN ubiquen a Nueva Delhi en el rol de balanceadora de la región. Al inaugurarse la Act East Policy, la Ministra de Relaciones Exteriores de India, Sushma Swaraj manifestó que "la asociación estratégica ASEANIndia debe su fuerza al hecho que nuestra 'Look East' hacia ASEAN se encuentra con su 'Look West' hacia India”' (Swaraj en Rajendram, 2014: 8). No obstante, Nueva Delhi se ha 
mantenido cautelosa, evitando proyectar una imagen que busque contener a China o de intromisión en la estrategia regional de su vecino más poderoso.

\section{Conclusiones}

India no ha contado con voluntad política para cumplir un rol regional importante y, en consecuencia, su política exterior careció de un diseño estratégico hacia el Sudeste Asiático durante gran parte de su vida independiente. Dicha limitación no sólo se debía a cuestiones internas del país -como las políticas económicas aislacionistas, el bajo nivel de crecimiento y desarrollo, los conflictos religiosos, etc.- sino que la dinámica propia de la Guerra Fría impidió una vinculación mayor.

Teniendo presente estos antecedentes, el objetivo del presente trabajo ha sido analizar la evolución de la Look East Policy lanzada por India a principios de los años noventa hasta llegar a la segunda década de los 2000 con una nueva designación, la Act East Policy. En este sentido, en un primer momento dicha orientación de política exterior fue menos un intento de la dirigencia india por darle a su país un protagonismo mayor en los asuntos regionales y más una respuesta a los cambios sistémicos y domésticos que afrontaba.

La LEP estuvo directamente vinculada a dos hechos de cabal relevancia. Por un lado, durante la Posguerra Fría India queda huérfana de su principal aliado, la Unión Soviética, lo cual redefinió su lugar en el mundo y en la región para salir de su aislamiento y adaptarse al nuevo sistema internacional. Por otro lado, la aplicación de políticas macroeconómicas neoliberales para liberalizar la economía tenía como objetivo principal superar los bajos niveles de crecimiento e integrar al país en el comercio internacional. Por ello, la búsqueda de nuevos socios comerciales se volvió de cabal importancia.

Ahora bien, los éxitos a nivel económico cosechados durante los años 90' supusieron para la elite política india un sentimiento de mayor confianza para disputar el protagonismo en la región así como a nivel internacional. En este sentido durante la Posguerra Fría ha afianzado su estatus de potencia media gracias su crecimiento económico acelerado, el desarrollo de su capacidad militar (y nuclear), los avances tecnológicos conseguidos y el progreso en el sector servicios. A dichas capacidades materiales se debe sumar el poder blando que ha construido en base a los ideales de los padres fundadores del país, es decir, la condición de ser la mayor democracia del mundo que fundamenta sus relaciones exteriores en los principios del no-alineamiento, la no interferencia en los asuntos internos y la cooperación internacional para lograr el beneficio mutuo. Las características mencionadas la han convertido en un socio difícil de rechazar.

En función de los cambios ocurridos a inicios de los noventa, la primer etapa de la LEP coincidió con el interés de la ASEAN de integrar a India a su estructura institucional. En otras palabras, el interés de vincularse fue recíproco. Y por esta razón es que India ascendió de categoría muy rápidamente hasta convertirse en un Socio de Diálogo Pleno para la Asociación, situación que le daba la potestad de participar de otros foros regionales nacidos en el núcleo de la ASEAN. No obstante su actuación no se restringió al ámbito de la Asociación sino que su ímpetu la llevó a crear varias organizaciones regionales para fomentar la cooperación y el comercio, involucrando a los países del SEA. Como ejemplos se puede mencionar el BIMSTEC y el MGC. 
La segunda etapa de la LEP, que coincidió temporalmente con el gobierno de Manmohan Singh, se caracterizó principalmente por profundizar la integración económica esta-blecida con la ASEAN. Tal es así que durante este período se alcanzó la firma del Tratado de Libre Comercio de Bienes, conocido como ASEAN+India, en el año 2009 y se hicieron grandes avances para la firma del Tratado de Libre Comercio de Servicios. También se die-ron avances de importancia en el ámbito de la defensa y la seguridad tanto a nivel intra-ASEAN como a nivel extra-ASEAN.

Desde el renombramiento de la LEP en AEP, el pretendido cambio cualitativo que el gobierno de Narendra Modi puso en marcha en cuanto a la política india hacia el Sudeste Asiático, se ha podido vislumbrar en los siguientes hechos. Efectivamente, ha ampliado el activismo político hacia prácticamente todos los estados de la región Asia-Pacífico, superando la perspectiva ASEAN-centrista que tuvieron los gobierno anteriores. Por otra parte, Modi también ha extendido las temáticas de vinculación con los países del Este de Asia, pero con una impronta mercantilista muy clara. Ello se encuentra ilustrado en la interconexión que existe entre el proyecto Make in India, las inversiones y la cooperación destinada para mejorar la conectividad física de India con la región, el propio comercio del sector armamentístico y el uso del discurso en los foros multilaterales en la defensa de la libre navegación.

No obstante, también es dable precisar que desde el surgimiento de la LEP hasta la actualidad, el tránsito realizado es de gran relevancia ya que los pasos que se están dando en la actualidad no serían posibles sin contar con la construcción de confianza en los más de veinte años transcurridos. Aunque se debe reconocer que el gobierno de Modi ha sabido capitalizar muy hábilmente los pasos dados en las décadas anteriores.

Resta indicar que la actual AEP afronta tres desafíos. En primer lugar, el ineludible ascenso de China y el rol protagónico que está teniendo en la región Asia-Pacífico, demostrado en las iniciativas de integración económica o financiera (se refiere a la Ruta de la Seda y al Banco Asiático de Infraestructura e Inversiones) que no sólo han captado la atención de los socios asiáticos sino también de los europeos. En segundo lugar, el triángulo estratégico India-China-Estados Unidos, al que se podría incluir a Japón, que en caso de desestabilización de tal sistema de interdependencia podría poner en vilo a todo el Sudeste Asiático y tener repercusiones globales. Por último, el manejo de la política interna de India será crucial para darle continuidad a la dirección de la política exterior para el Este de Asia, que se podría denominar que se trata de una política de Estado.

\section{Bibliografía}

Babu, Rajesh (2013) "India-ASEAN Free Trade Agreement: Ramifications for India”, Asian Journal of WTO \& International Health Law and Policy, Taiwan, v. 8, n², pp. 461-481.

Banco Interamericano de Desarrollo (BID) (2010) "India: oportunidades y desafíos para

América Latina", [coordinación y redacción de Mauricio Mesquita Moreira] http://www.iadb.org/intal/intalcdi/PE/2010/05898.pdf. . 
Bhatia, Rajiv (13/01/2016) "Act East Policy. Emerging Contours", Observer Research Foundation, New Delhi, India http://www.orfonline.org/research/act-eastpolicy-emerging-contours-2/

Discurso del Ministro de Relaciones Exteriores Shri Yashwant Sinha en la Universidad de Harvard, 29 de septiembre de 2003. Disponible en:

http://mea.gov.in/Speeches-

State-

ments.htm?dtl/4744/Speech+by+External+Affairs+Minister+Shri+Yashwant+Sin ha+at+Harvard+University

Dussort, María Noel (2013) “¿Por qué India pasó a ser un consumidor energético global? Un análisis sobre el contexto energético interno del gigante asiático", Revista Cadernos Argentina-Brasil, Rio de Janeiro, v. 2, n 1, pp. 47-60 http://www.epublicacoes.uerj.br/index.php/neiba/article/view/8251

Efstathopoulos, Charalampos (2011) "Reinterpreting India's Rise through the Middle Power Prism", Asian Journal of Political Science, Singapore, v. 19, $n^{\circ} 1$, pp. 7495. doi:10.1080/02185377.2011.568246

Frazier, Mark W. (2000) China-India relations since Pokhran II: assessing sources of conflict and cooperation, Access Asia Review, Vol. 3, № 2, The National Bureau of Asian Research.

Giridharadas, Anand (10/01/2009) "India Has a Soft Spot for Bush", The New York Times. Disponible en: http://www.nytimes.com/2009/01/11/weekinreview/11giridharadas.html

González Castañeda, Mario (2010) “La ciencia, la tecnología y los átomos en el contexto de las relaciones India-China: fetiche y tabú", Estudios de Asia y África, vol. XLV, no 1 , pp. 69-115.

Gupta, Ranjit (2007) “India's 'Look East Policy”” en Atish Sinha y Madhup Mohta (eds.), India's Foreign Policy. Challenges and Opportunities, New Delhi: Academic Foundation, pp. 351-382.

"India, Pakistan become full SCO members" (11/07/2015) The Hindu, New Delhi. Disponible en: http://www.thehindu.com/news/international/india-gets-fullmembership-of-the-shanghai-cooperation-organisation-along-withpakistan/article7407873.ece

Jyoti (2013) "India's Look East Policy: In its Second Phase", Global Journal of Pharmaceutical Sciences and Education, New Delhi, v. 2, $n^{\circ} 1$, pp. 1-14. http://www.ripublication.com/gjpse.htm

Lara, Ignacio F. (2012), "Potencialidades y límites de Brasil como potencia media emergente", Anuario Americanista Europeo, Paris, $n^{\circ}$ 10, pp. 53-72.

Make in India (2017) "2 years of Make in India". https://drive.google.com/file/d/OBTv7_upCKANcjZUR2pVSOdQaHc/view

Mato Bouzas, Antía (2009) "La política exterior india: las dimensiones global y regional”, Real Instituto Elcano, Madrid, documento de trabajo, pp. 1-28 
http://www.realinstitutoelcano.org/wps/portal/rielcano/contenido?WCM_GLO BAL_CONTEXT=/elcano/elcano_es/zonas_es/asia-pacifico/dt27-2009

Mato Bouzas, Antía (2007) "La política de India hacia Myanmar: la importancia de las relaciones de vecindad en Asia meridional”, Real Instituto Elcano, Madrid.

Ministerio de Asuntos Exteriores de India (2017) "ASEAN-India Relations". http://www.mea.gov.in/aseanindia/20-years.htm

Ministerio de Comercio e Industria de India (2016) "Estadísticas comerciales". http://commerce.nic.in/MOC/index.asp

Modi, Narendra (21/11/2015) "Text of the Opening Statement by the PM at the ASEANIndia Summit", Press Information Bureau, Government of India Prime Minister's Office. Disponible en: http://pib.nic.in/newsite/PrintRelease.aspx?relid=131761

Modi, Narendra (12/11/2014) "PM's speech at the 12th India-ASEAN Summit, Nay Pyi Taw, Myanmar" http://www.narendramodi.in/remarks-by-the-pm-at-the-12thindia-asean-summit-nay-pyi-taw-myanmar-2

Mohapatra, Biswajit (2014) "India \& South East Asia. Expanding Opportunities for Regional Integration and India's Look East Policy" en Mohammad Badrul Alam (ed.) Contours of Indian Foreign Policy-Changes and Challenges, New Delhi: Reference Press Publications, pp. 186-201.

Muni, S. D. (2011) “India's 'Look East' Policy: The Strategic Dimension”, Institute of South Asian Studies (ISAS), Singapore, Working Paper, v. 1, $\mathrm{n}^{\circ} 121, \mathrm{pp} .1-25$. https://www.hitpages.com/doc/6668425011134464/2\#pageTop

"Myanmar's landmark elections explained" (03/12/2015), BBC, London, United Kingdom Disponible en: http://www.bbc.com/news/world-asia-33547036

Naidu, G. V. C. (2013) "India and East Asia: The Look East Policy", Perceptions, Ankara, Turkey, v. 18, n 1, pp. 53-74. http://sam.gov.tr/wpcontent/uploads/2013/06/GVC_Naidu.pdf

Nye, Joseph (2004) Soft Power. The Means to Success in the Wolrld Politics. New York: Public Affairs.

Parameswaran, Prashanth (17/11/2014) “Modi Unveils India's 'Act East Policy' to ASEAN in Myanmar", The Diplomat, Tokyo, Japan http://thediplomat.com/2014/11/modi-unveils-indias-act-east-policy-to-aseanin-myanmar/

Rajan, Rajiv (2016) “PM Modi's Sojourn to South East Asia: Reinvigorating the Partnership in the New Millennium", Issue brief, Indian Council of World Affairs, New Delhi.

Rajan, Ramkishen S. y Rahul Sen (2000) "Trade Reforms In India Ten Years On: How Has It Fared Compared To Its East Asian Neighbours?", Center for International Economic Studies, Adelaide, Australia, discussion paper $n^{\circ} 147$. https://www.adelaide.edu.au/cies/documents/papers/0147.pdf

Rajendram, Danielle (2014) "India's new Asia-Pacific strategy: Modi acts East", The Lowy Institute for International Policy, Sidney, Australia 
http://www.lowyinstitute.org/files/indias-new-asia-pacific-strategy-modi-actseast.pdf

Roychoudhury, Supriya; CHENOY, Anuradha; CHOPRA, Deepta and JOSHI, Anuradha (2015) "Is Indian Development Cooperation Taking a New Direction Under Modi?", Rising Powers in International Development Programme, IDS Policy briefing, Issue 94, Institute of Development Studies, Brighton.

"SAARC Development Bank averted" (21/08/2015), Bhutan News Network. Disponible en: http://www.bhutannewsnetwork.com/2015/08/saarc-development-bankaverted/

Sahni, Varun (2013) "India: a pesar de sus limitaciones, una potencia emergente", Nueva Sociedad, $\mathrm{N}^{\circ} 246$, julio-agosto de 2013, pp. 102-114.

Sidhu, W.P.S y Godbole, Shruti (2015) "Bold Initiatives Stymied by Systemic Weakness", en, Brookings India (Eds.) Modi's Foreign Policy @365: Course Correction, Nueva Delhi, pp. 3-4 https://www.brookings.edu/opinions/modis-foreign-policy365-course-correction/

Sikri, Rajiv (2009) “India's Look East Policy: A Critical Assessment. Interview with Amb. Rajiv Sikri", Institute of Peace and Conflict Studies Special Report, octubre, Nueva Delhi, India http://www.ipcs.org/special-report/india/indias-look-east-policya-critical-assessment-85.html

Singh, Udai Bhanu (1999) "India and the ARF: the post-Pokhran II phase", Strategic Analysis, v. 22, no 10, pp. 1591-1606. Disponible en: https://www.idsa-india.org/anjan9-10.html

Tharoor, Shashi (2012), Pax Indica, India and the World of the Twenty-first Century, New Delhi: Pinguin Books.

"U.S. imposes sanctions on India" (13/05/1998) CNN. Disponible en: http://edition.cnn.com/WORLD/asiapcf/9805/13/india.us/

Vieytes, Rut (2004), Metodología de la investigación en organizaciones, mercado y sociedad: epistemología y técnicas, Buenos Aires: De las ciencias. 